\title{
Implementation of Automated Blood Culture With Quality Assurance in a Resource-Limited Setting
}

\author{
Anja von Laer ${ }^{1,2,3 *}$, Micheline Ahou N'Guessan ${ }^{4}$, Fidèle Sounan Touré ${ }^{4}$, Kathrin Nowak ${ }^{2}$, \\ Karin Groeschner ${ }^{2}$, Ralf Ignatius ${ }^{5}$, Johannes Friesen ${ }^{5}$, Sara Tomczyk ${ }^{2}$, \\ Fabian H. Leendertz ${ }^{6}$, Tim Eckmanns ${ }^{2}$ and Chantal Akoua-Koffi ${ }^{4}$ \\ ${ }^{1}$ Postgraduate Training for Applied Epidemiology, Department of Infectious Disease Epidemiology, Robert Koch Institute, \\ Berlin, Germany, ${ }^{2}$ Department of Infectious Disease Epidemiology, Robert Koch Institute, Berlin, Germany, ${ }^{3}$ European \\ Programme for Intervention Epidemiology Training, European Centre for Disease Prevention and Control, Stockholm, \\ Sweden, ${ }^{4}$ Laboratoire Central, Centre Hospitalier et Universitaire de Bouaké, Bouaké, Côte d'Ivoire, ${ }^{5}$ Department of \\ Microbiology, Medical Care Unit Labor $28 \mathrm{GmbH}$, Berlin, Germany, ${ }^{6}$ Research Group Epidemiology of Highly Pathogenic \\ Microorganisms, Robert Koch Institute, Berlin, Germany
}

OPEN ACCESS

Edited by:

Chantal M. Morel, Université de Genève, Switzerland

Reviewed by: James Fackler Johns Hopkins University,

United States

Frieder Schaumburg, University of Münster, Germany

Olga Perovic,

National Institute of Communicable

Diseases (NICD), South Africa

${ }^{*}$ Correspondence: Anja von Laer laera@rki.de

Specialty section

This article was submitted to Infectious Diseases - Surveillance,

Prevention and Treatment, a section of the journal

Frontiers in Medicine

Received: 09 November 2020 Accepted: 23 April 2021 Published: 21 May 2021

Citation: von Laer A, N'Guessan MA, Touré FS, Nowak $K$, Groeschner $K$, Ignatius $R$, Friesen J, Tomczyk S, Leendertz FH,

Eckmanns T and Akoua-Koffi C (2021) Implementation of Automated Blood Culture With Quality Assurance in a

Resource-Limited Setting.

Front. Med. 8:627513.

doi: 10.3389/fmed.2021.627513
Background: Blood cultures (BC) have a high clinical relevance and are a priority specimen for surveillance of antimicrobial resistance. Manual BC are still most frequently used in resource-limited settings. Data on automated BC performance in Africa are scarce. We implemented automated BC at a surveillance site of the African Network for improved Diagnostics, Epidemiology and Management of Common Infectious Agents (ANDEMIA).

Methods: Between June 2017 and January 2018, pairs of automated BC (BacT/ALERT ${ }^{\circledR}$ FA Plus) and manual BC (brain-heart infusion broth) were compared at a University hospital in Bouaké, Côte d'Ivoire. BC were inoculated each with a target blood volume of $10 \mathrm{ml}$ from the same venipuncture. Automated BC were incubated for up to 5 days, manual $\mathrm{BC}$ for up to 10 days. Terminal subcultures were performed for manual BC only. The two systems were compared regarding yield, contamination, and turnaround time. For quality assurance, isolates were retested in a German routine microbiological laboratory.

Results: $\mathrm{BC}$ sampling was increased from on average $24 \mathrm{BC}$ to $63 \mathrm{BC}$ per month. A total of 337 matched pairs of BC were included. Automated BC was positive in $36.5 \%$, manual BC in $24.0 \%$ (p-value < 0.01 ), proportion of contamination was 47.9 and $43.8 \%$, respectively $(p$-value $=1.0)$. Turnaround time of positive $B C$ was shortened by 2.5 days with automated compared to manual BC $(p<0.01)$. Most common detected pathogens in both systems were Klebsiella spp. (26.0\%) and Staphylococcus aureus (18.2\%). Most contaminants were members of the skin flora. Retesting of 162 isolates was concordant in $79.6 \%$ on family level.

Conclusions: Implementing automated BC in a resource-limited setting is possible and improves microbiological diagnostic performance. Automated BC increased yield and shortened turnaround times. Regular training and mentorship of clinicians has to be intensified to increase number and quality of BC. Pre-analytical training to improve diagnostic stewardship is essential when implementing a new microbiological method. 
Retesting highlighted that manual identification and antimicrobial susceptibility testing can be of good quality and sustainable. The implementation of automated tools should be decided individually according to economic considerations, number of samples, stable supply chain of consumables, and technical sustainability.

Keywords: blood culture, sub-Saharan Africa, bacterial infection, laboratory automation, quality control

\section{INTRODUCTION}

In low- and middle-income countries (LMIC), infectious diseases remain one of the main causes of morbidity and mortality. Febrile illness is a leading cause for admission to hospitals in Africa (1). Despite the major burden of infectious diseases, the availability of diagnostic microbiology services for bloodstream infections other than malaria is often limited by cost, infrastructure, and personnel constraints. Antimicrobial resistance (AMR) compromises the outcome of bloodstream infections and reduces treatment options. Although reliable data on AMR in Africa is scarce according to the World Health Organization (WHO), the available data indicate that AMR is increasing in Africa (2). Furthermore, second-line drugs to treat infections with resistant bacteria are not easily accessible in all countries. One of the five objectives of the WHO Global Action Plan on AMR is to strengthen the knowledge and evidence base through surveillance and research (3). WHO defines blood cultures (BC) as a priority specimen for AMR surveillance and it is recommended to prioritize key clinical specimens in resource-limited settings $(4,5)$. WHO also highlights diagnostic stewardship as an integral part to build up AMR surveillance systems, defining this as the "coordinated guidance and interventions to improve appropriate use of microbiological diagnostics to guide therapeutic decisions" (6).

During the last two decades, automated systems with computer-driven monitor of $\mathrm{CO}_{2}$ concentration for the detection of microorganisms in $\mathrm{BC}$ have been developed. Comparisons of automated $\mathrm{BC}$ systems to conventional manual procedures have shown a higher sensitivity and a shorter time to positivity (79). As resources are limited, manual $\mathrm{BC}$ is often used in African countries $(10,11)$. Data on automated BC implementation in resource-limited settings, especially in sub-Saharan Africa, are scarce $(11,12)$.

To combat infectious diseases including AMR, a surveillance system has been developed at sentinel sites in Côte d'Ivoire among other West African countries as part of the African Network for improved Diagnostics, Epidemiology and Management of Common Infectious Agents (ANDEMIA, https://www.andemia.org) (13).

Bouaké, the second largest city in Côte d'Ivoire, is located in the central part of the country 350 kilometers north of Abidjan, the economic and political capital and has a population of $\sim 700,000$ inhabitants. Bouaké was the center of military and political crises in Côte d'Ivoire, particularly from 1999 to 2011. The University Hospital Bouaké (CHU-B), the country's only academic hospital outside Abidjan, had to suspend all except the most basic medical services between 2002 and 2011. CHU-B today has 268 beds, 27,189 admissions and 62,515 consultations in 21 departments per year.

In 2012-2014, a study on manual BC from severely ill patients at CHU-B showed a positivity rate of $22.5 \%$ with highest rates in pediatrics (14). The most commonly isolated pathogen was Klebsiella pneumoniae, followed by Salmonella enterica. In 2017, a study on the resistance patterns of Klebsiella pneumoniae from clinical samples at CHU-B showed that most isolates derived from $\mathrm{BC}$ and that $84 \%$ produced extended spectrum beta-lactamases (ESBL) (15). Routine BC sampling might be hampered by shortage of resources and empiric antibiotic treatment is often started without diagnostics. In 2016, on average $24 \mathrm{BC}$ were processed per month (16). To improve diagnostics and to support culture-guided therapy, an automated $\mathrm{BC}$ system was implemented at CHU-B in the frame of the ANDEMIA project.

The aim of the present study was to verify automated $\mathrm{BC}$ in the laboratory, to compare automated $\mathrm{BC}$ with manual $\mathrm{BC}$ regarding proportion positive, proportion contaminated and turnaroundtimes and to improve $\mathrm{BC}$ sampling in order to improve patient care as well as AMR surveillance.

\section{METHODS}

We conducted a prospective laboratory study from 28 June 2017 to 18 January 2018 to implement automated BC in a resourcelimited setting. The implementation was accompanied by a 6week on-site mentorship period of a clinical microbiologist from Germany and 1 week of theoretical and practical training on BC processing and antimicrobial susceptibility testing (AST) with an international team of experts from Burkina Faso, Côte d'Ivoire, the Democratic Republic of Congo and Germany.

\section{Participants}

$\mathrm{BC}$ from consecutive patients admitted to any department at CHU-B with self-reported fever or fever on admission $\left(\geq 38.0^{\circ} \mathrm{C}\right)$ and suspected bloodstream infection were included in the study. No exclusion criteria were applied. The decision to take blood samples for culture rested solely on the physicians' clinical judgement. The study was imbedded in the routine workup.

\section{Training of Clinicians}

The departments were informed about the study by the head of the laboratory on 26 June 2017. Clinicians were instructed to take a set of $\mathrm{BC}$ for automated and manual processing from each patient above 2 years of age with an indication for $\mathrm{BC}$ sampling. Training of clinicians on good practice for $\mathrm{BC}$ sampling was 
performed in a one-hour session by laboratory staff in the three departments that have sent most BC. One-page job aids on how to take a set of $\mathrm{BC}$ for children and adults were distributed to each department in the hospital.

\section{Blood Culture Processing}

Automated BC were processed using the BacT/ALERT ${ }^{\circledR} 3 \mathrm{D}$ system (bioMérieux, Marcy L'Etoile, France). Commercially produced BacT/ALERT ${ }^{\circledR}$ FA bottles were used for adults above 15 years of age and BacT/ALERT ${ }^{\circledR}$ PF bottles were used for children below 15 years of age. In children when insufficient blood volume was obtained and who were below three years of age, only the BacT/ALERT ${ }^{\circledR}$ PF bottle was inoculated. For manual BC, commercially available BC was used for adults and children (HIMEDIA HiCombiTM Dual Performance Medium and HIMEDIA BHI Broth - Supplemented x/0.05\% SPS, HIMEDIA Laboratories, Mumbai, India). In case of stockout, self-prepared brain-heart infusion (BHI) broth $(60 \mathrm{ml}$ for adult and $30 \mathrm{ml}$ for pediatric bottles) in suitable glass bottles was used instead. Commercially available BC media had to be used as suitable glass bottles to be filled manually were not sufficiently available for the increased number of samples. Clinicians were asked to take $20 \mathrm{ml}$ blood from adults and $10 \mathrm{ml}$ from children above 2 years via syringe, and to fill a pair of BC bottles (i.e., $10 \mathrm{ml}$ each in adults and 3-4 and 5$6 \mathrm{ml}$ for children for automated and manual BC, respectively, according to manufacturer's instructions). As weighing each bottle before and after blood sampling to measure the exact volume of inoculated blood was not feasible in the daily routine and would have delayed $\mathrm{BC}$ processing, different types of $\mathrm{BC}$ bottles were weighed beforehand. Each BC bottle was weighed after processed in the laboratory to calculate the inoculated blood volume.

Both BC systems were processed according to the newly revised Standard Operating Procedures (SOPs) in the laboratory. Automated BC bottles were incubated for up to 5 days according to manufacturer's instructions. Manual BC bottles were incubated at $35 \pm 2{ }^{\circ} \mathrm{C}$ for up to 10 days with final subculture and checked daily for turbidity or other signs of growth (e.g., haemolysis, pellicle formation on surface or gas production). Positive BC were inoculated onto locally produced 5\% columbia blood agar, chocolate agar and Hektoen enteric agar, dependent on the result of the Gram stain, and incubated at $35 \pm 2{ }^{\circ} \mathrm{C}$ in ambient or $\mathrm{CO}_{2}$-enriched air (candle jar) for $24-48 \mathrm{~h}$ (Bio-Rad Laboratories Ltd., Hercules, United States of America and Oxoid Ltd., Hampshire, United Kingdom). Identification of isolates was done by cultural morphology, biochemical and antigenic methods (Supplementary Figure 1). Gram stain was performed from positive $\mathrm{BC}$ and from colonies on agar. Antimicrobial susceptibility testing (AST) was done for isolated pathogenic bacteria by Kirby-Bauer disc diffusion test using references from the European Committee on Antimicrobial Susceptibility Testing (EUCAST) (17). If no breakpoint for disc diffusion was available for EUCAST (e.g., vancomycin for staphylococci), the reference from the Clinical \& Laboratory Standards Institute (CLSI M100, 31 st edition) was used instead.

\section{Data Collection}

The routine laboratory request form for microbiological investigation for BC provided to clinicians was adapted to include information on demographics, time of sampling and antibiotic treatment. The attending microbiologist defined the respective recovered isolate as contaminant or pathogen individually based on multiple criteria including identification (e.g., common skin contaminant), number of positive $\mathrm{BC}$ and clinical information. A positive $\mathrm{BC}$ was defined as a $\mathrm{BC}$ with subsequent growth of microorganisms.

The proportion of positive $\mathrm{BC}$ was defined as the number of positive $\mathrm{BC}$ divided by the number of all processed $\mathrm{BC}$.

The proportion of contaminated $\mathrm{BC}$ was defined as the number of contaminated positive $\mathrm{BC}$ divided by the number of positive BC.

The proportion of $\mathrm{BC}$ with a false-positive signal was defined as the number of $\mathrm{BC}$ that had a positive signal without growth in subculture divided by the number of $\mathrm{BC}$ with that respective information.

The turnaround-time was measured as the days from BC sampling until the final microbiological result was available in the laboratory.

The time-to-detection was measured as the hours from loading the $\mathrm{BC}$ bottle into the machine or incubator until the $\mathrm{BC}$ was flagged positive.

The time-to-analysis was measured as the hours from loading the $\mathrm{BC}$ bottle into the machine or incubator until the positive $\mathrm{BC}$ bottle was removed and processed.

The time-to-positivity was measured as the hours from BC sampling until the $\mathrm{BC}$ was flagged positive.

\section{Quality Assurance/Retesting of Isolates}

Since CHU-B did not have an active external quality assurance programme, an inter-laboratory comparison was done and isolates were retested in a German routine microbiological laboratory.

Bacterial and fungal isolates, irrespective if they were isolated from automated and/or manual BC, were stored in ESwabs (Copan, Brescia, Italy) at $4^{\circ} \mathrm{C}$ until the end of the study and then shipped to Berlin, Germany. They were revived on blood agar and checked for purity. If multiple strains were isolated from one $\mathrm{BC}$, all strains were retested, accordingly. Microorganisms were identified using matrix-assisted laser desorption ionization time-of-flight mass spectrometry (MALDI-TOF MS, Bruker Daltonik, Bremen, Germany; software: MBT 7854 MSP Library, 2018) or Vitek ${ }^{\circledR} 2$ (bioMérieux, Marcy L'Etoile, France; software version: 8.01, 2018). AST was performed using Vitek ${ }^{\circledR} 2$ or disc diffusion using references from EUCAST (18). Isolates identified as Salmonella Typhi at CHU-B were excluded from retesting in Germany for safety reasons as handling is recommended under higher safety levels. Comparison of identification of isolates was done stepwise regarding family/group, genus, and species level. If species level could not be determined using conventional methods (e.g., for nonfermenters or coagulase-negative staphylococci), genus or group level was counted. Family or group were defined as follows: Non-fermenter, Enterobacterales, gram-positive rods (Bacillus 
spp.), Micrococcaceae/Staphylococcus spp., fungi, Enterococcus spp./Streptococcus spp. Genus was defined as follows: Nonfermenter (other than Pseudomonas spp.), Escherichia spp., Alcaligenes spp., Bacillus spp., Citrobacter spp., Enterobacter spp., Enterococcus spp., Klebsiella spp., yeasts, Micrococcus spp., Pseudomonas spp., Salmonella spp., Staphylococcus spp., Streptococcus spp., Stenotrophomonas spp. and Kocuria spp.

\section{Data Analysis}

All data were double-entered into the EpiData software (EpiData Association, Odense, Denmark) (19). Analyses were conducted using Stata version 15.1 (StataCorp, Texas, USA) and Microsoft Excel (2010). Automated and manual BC were compared in terms of proportion positive, proportion contaminated, turnaround-times, recovery of different bacteria and maintenance. If automated or manual BC bottles were received in the laboratory only with no matching pair, they were excluded from the comparison. Statistical differences between automated and manual BC were calculated using McNemars exact test, to compare medians the Wilcoxon signed-rank test was used. A p-value below 0.05 was defined as statistically significant. As different manual BC bottles had to be used, a subgroup analysis of proportion positive and contaminated in different bottles was performed using Fisher's exact test. Interpretations of AST from CHU-B and Germany were compared. If duplicates from automated and manual $\mathrm{BC}$ were re-tested, only one isolate was included in the analysis. If the interpretation of AST for the same species was different in automated and manual BC, the more resistant test was included in the analysis.

\section{Ethics}

The studies involving human participants were reviewed and approved by Charité University Ethics Committee Berlin, Germany (reference number: EA2/230/17) and the National Ethics Committee for Research in Abidjan, Côte d'Ivoire (reference number: 141/MHSP/CNER-km). Isolates were obtained through the hospitals routine diagnostic and used for this study.

\section{RESULTS}

\section{Comparison of Automated and Manual BC}

In total, $440 \mathrm{BC}$ were sent for analysis during the study period of 7 months. BC sampling was increased from on average 24 $\mathrm{BC}$ to $63 \mathrm{BC}$ per month. Of those, 103 automated $\mathrm{BC}$ arrived without corresponding manual $\mathrm{BC}$ in the laboratory ( 90 pediatric and 13 adult $\mathrm{BC}$ ) and were subsequently removed from the final analysis. In total, 337 pairs of automated and manual BC were included in the analysis. The median age of patients was 31 years (range: $0-88$ years). Of 333 patients with information on sex, $53.8 \%$ of patients were male $(n=179)$. Of 330 patients with information on antibiotic treatment, 191 (57.9\%) had received antibiotics before BC sampling with a median of 3 days (range: 0 21 days). The three most common administered antibiotics were ceftriaxone, amoxicillin and clavulanic acid, and gentamycin.

Volumes of BC bottles varied, but were similar in automated and manual BC. Median volume in adult BC was $10.0 \mathrm{ml}$ and in pediatric $\mathrm{BC} 3.6$ and $3.0 \mathrm{ml}$ in automated and manual $\mathrm{BC}$, respectively.

Of 337 pairs, the results of 259 (76.9\%) manual and automated BC were concordant (Table 1). Of those, 196 were negative (75.7\%), whereas 63 were positive (24.3\%). In 78 discordant pairs (23.1\%), 18 BC showed growth only in manual BC (23.1\%) and 60 only in automated BC $(76.9 \%)$.

The proportion of positive $\mathrm{BC}$ was significantly higher in automated BC $(n=123,36.5 \%)$ than in manual BC $(n=81$, $24.0 \%, p<0.01$, Table 2 ). The proportion of contaminated BC was not significantly higher in automated BC $(n=58,47.5 \%)$ than in manual BC $(n=35,43.2 \%, p=1.0)$. The proportion of $\mathrm{BC}$ that show growth with a pathogen was $23.3 \%$ in automated BC and $15.2 \%$ in manual BC.

Subgroup analysis of different types of manual BC bottles (commercially available vs. self-produced BC) regarding proportion positive and proportion contaminated did not show a significant difference (data not shown).

Automated BC showed significantly less frequently a false positive signal: whereas $40.2 \%(n=88)$ of manual BC signaled positive, but no microorganisms grew in sub-culture, 5.6\% $(n=$ $10, p<0.01$ ) of automated BC showed a false positive signal. The median inoculated blood volume in these false positive BC was $10 \mathrm{ml}$.

Turnaround times were shorter with automated BC than with manual $\mathrm{BC}$ irrespective of the result of the $\mathrm{BC}(p<0.01$, Table 2$)$. The turnaround time from $\mathrm{BC}$ sampling until final results was 6.0 days shorter with automated $\mathrm{BC}$ than with manual $\mathrm{BC}$. The time from loading the $\mathrm{BC}$ bottle into the machine or incubator until the positive $\mathrm{BC}$ was removed and analysis was started was shortened by 3 days $(69.6 \mathrm{~h}$ ) with automated BC compared to manual BC. For positive $\mathrm{BC}$ the turnaround time from $\mathrm{BC}$ sampling until final result was shortened with automated $B C$ by 2.5 days and the time from incubating the $\mathrm{BC}$ bottle until the start of analysis by $76.4 \mathrm{~h}$ (data not shown).

The most often detected bacteria in automated and manual BC were coagulase-negative staphylococci ( $n=56 / 152,36.8 \%$ ); all of those were interpreted as contaminants (Figure 1). The most often detected pathogens were Klebsiella spp. $(n=$ 20/152, 13.2\%), Staphyloccoccus aureus $(n=14 / 152,9.2 \%)$ and Enterobacter spp. $(n=10 / 152,6.6 \%)$. The detection rate was higher with automated $\mathrm{BC}$ for almost all detected bacteria.

If automated $\mathrm{BC}$ and manual $\mathrm{BC}$ were both positive $(n=$ $63)$, the same microorganism was identified in $82.5 \%(n=52$, Figure 2). Of those, 30 microorganisms (57.7\%) were interpreted as pathogens. For $83.3 \%$ of those pathogens $(n=25)$, results

TABLE 1 | Summary of concordant and discordant pairs of automated BC and manual BC, CHU-B, Côte d'Ivoire 2017-2018, N = 337.

\begin{tabular}{lccc}
\hline Results Manual BC & \multicolumn{3}{c}{ Results Automated BC } \\
\cline { 2 - 4 } & Negative & Positive & Total \\
\hline Negative, $n(\%)$ & $196(58.2 \%)$ & $60(17.8 \%)$ & $256(76.0 \%)$ \\
Positive, $n(\%)$ & $18(5.3 \%)$ & $63(18.7 \%)$ & $81(24.0 \%)$ \\
Total, $n(\%)$ & $214(63.5 \%)$ & $123(36.5 \%)$ & $337(100 \%)$
\end{tabular}




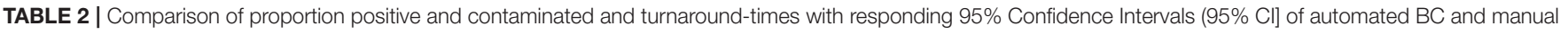
BC, CHU-B, Côte d'Ivoire 2017-2018, N = 337 .

\begin{tabular}{|c|c|c|c|c|c|c|c|c|}
\hline \multirow[t]{2}{*}{ Variables } & \multicolumn{3}{|c|}{ Automated BC } & \multicolumn{3}{|c|}{ Manual BC } & \multirow[t]{2}{*}{ Difference } & \multirow[t]{2}{*}{$p$-value } \\
\hline & $n$ & Value & (95\% Cl) & $n$ & Value & $(95 \% \mathrm{Cl})$ & & \\
\hline Proportion positive in \% & 123 & 36.5 & (31.3-41.9) & 81 & 24.0 & (19.6-29.0) & 12.5 & $<0.01$ \\
\hline Proportion contaminated in \% & 58 & 47.9 & $(38.8-57.2)$ & 35 & 43.8 & $(32.7-55.3)$ & 4.1 & 1.0 \\
\hline median TAT $^{\mathbf{1}}$ (turnaround-time) in days & 332 & 5.0 & $(5.0-5.0)$ & 331 & 11.0 & $(11.0-12.0)$ & 6.0 & $<0.01$ \\
\hline median $\mathbf{T T P}^{2}$ (time-to-positivity) in hours & 112 & 19.7 & $(17.6-22.1)$ & 69 & 69.2 & $(45.3-113.6)$ & 49.5 & $<0.01$ \\
\hline median $\mathbf{T T D}^{3}$ (time-to-detection) in hours & 126 & 17.0 & $(15.6-19.2)$ & 80 & 90.4 & $(59.0-11.7)$ & 73.4 & $<0.01$ \\
\hline median TTA 4 (time-to-analysis) in hours & 127 & 26.8 & $(25.2-28.0)$ & 79 & 96.4 & $(69.2-117.0)$ & 69.6 & $<0.01$ \\
\hline
\end{tabular}

${ }^{1}$ TAT: BC sampling until final result.

${ }^{2}$ TTP: BC sampling until flagged positive.

${ }^{3}$ TTD: Loading in BC machine/incubator until flagged positive.

${ }^{4}$ TTA: Loading in BC machine/incubator until removal and initial work.

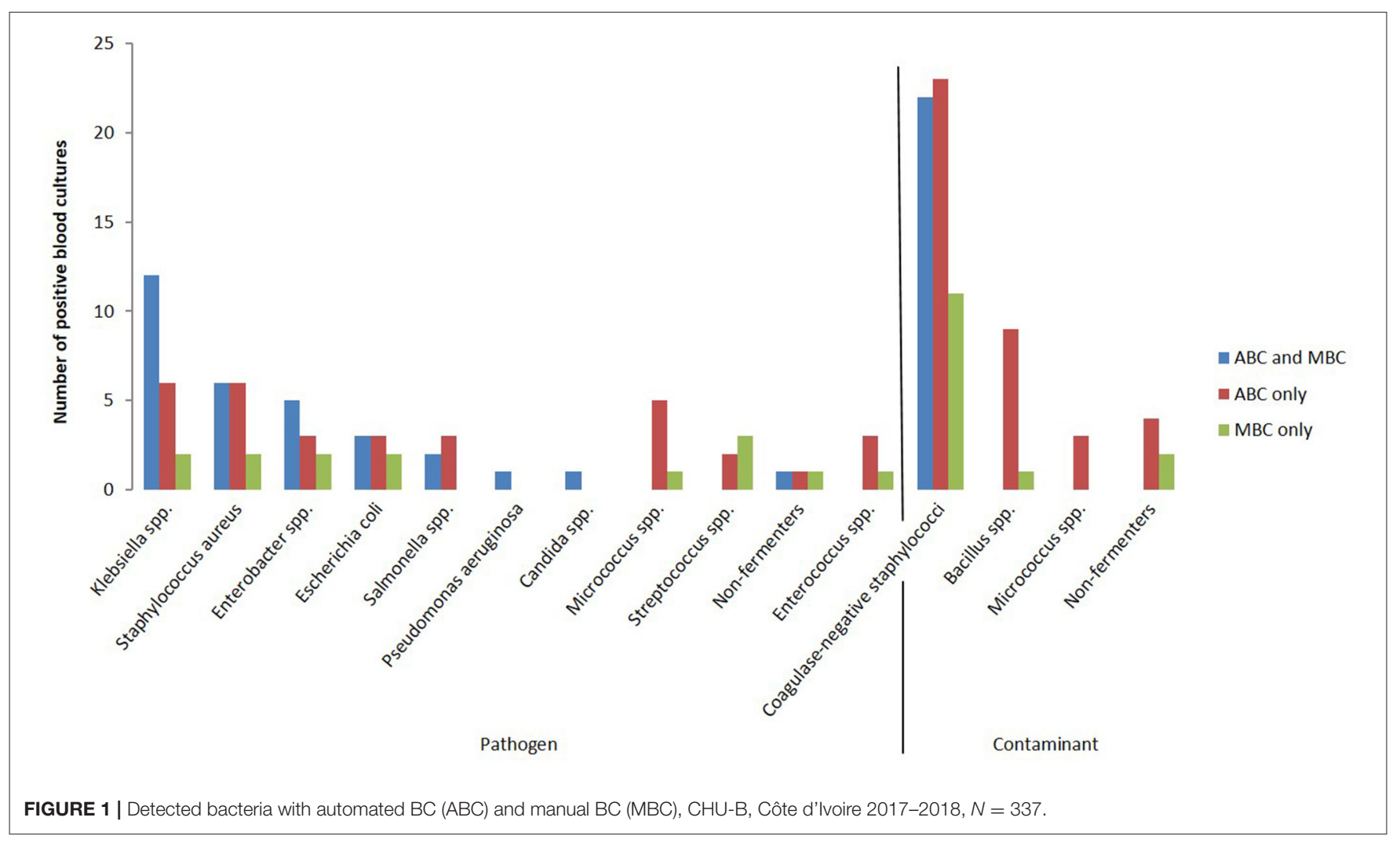

of antimicrobial susceptibility testing (AST) were available from both, automated and manual BC including 19 Enterobacterales and six Staphylococcus aureus isolates. Clinical interpretation of AST results in automated and manual BC were concordant in $52.0 \%(n=13)$; in $48.0 \%(n=12)$, the AST of at least one antibiotic was interpreted differently in automated and manual $\mathrm{BC}$ for the same pathogen.

The subgroup analysis of discordant pairs, where the automated $\mathrm{BC}$ was positive and the final result of the corresponding manual $\mathrm{BC}$ was negative, revealed that in $6.7 \%$ (4/60), the manual BC was false-positive, i.e., the BC was signaled positive, but no microorganisms were detected in Gram stain or subculture. In $11.1 \%(2 / 18)$ of discordant pairs in which the final result of the manual $\mathrm{BC}$ was positive but the automated $\mathrm{BC}$ was negative, the automated BC bottle was false-positive. In discordant pairs, the median inoculated blood volume was higher in manual BC than in automated BC. Adult automated BC bottles were filled with a median of $9.5 \mathrm{ml}$ and manual $\mathrm{BC}$ with $10.0 \mathrm{ml}$; pediatric automated $\mathrm{BC}$ bottles were filled with a median of $3.2 \mathrm{ml}$ and manual BC with $9.4 \mathrm{ml}$.

Initial and maintenance requirements for the automated and commercially available manual BC systems varied (Table 3 ). In particular, the hands-on time was reduced with automated $\mathrm{BC}$ compared to manual BC. The expenses for BC bottles were also 


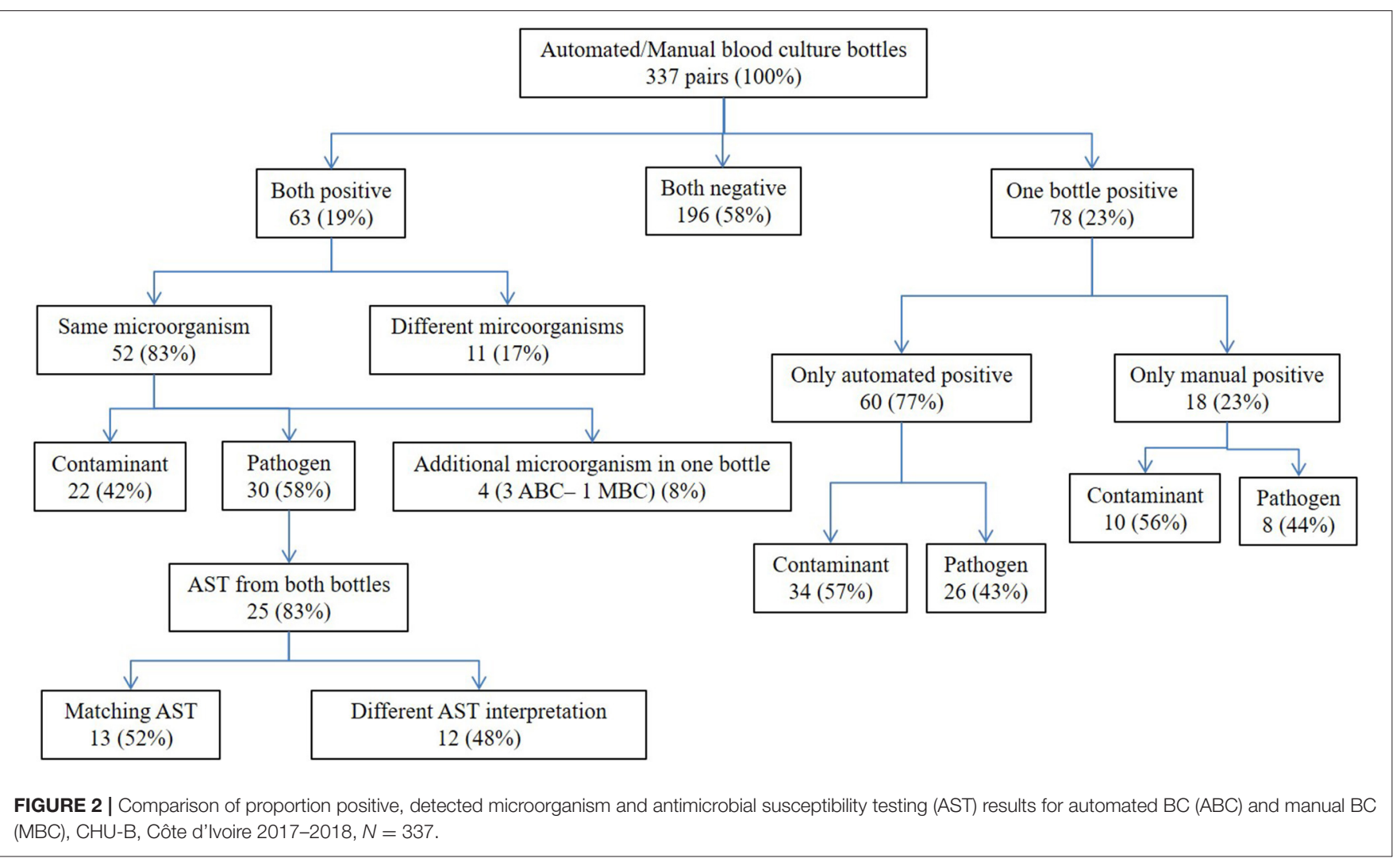

TABLE 3 | Comparison of initial and maintenance requirements for automated and manual BC, CHU-B, Côte d'Ivoire 2017-2018.

\begin{tabular}{lll}
\hline & Automated BC & Manual BC \\
\hline Costs of machine & - Initial funding necessary & - Incubator was available \\
Costs of BC bottles & - Similar if commercially available manual BC bottles are used & \\
Training & - Intensive training and continuous mentorship of all & - Staff were already trained in manual BC \\
& laboratory staff required & - Additional training on ventilation of bottles \\
& - Delayed incubation if staff during weekend or night shifts & - Incubation of BC bottles could be started throughout the \\
Maintenance & - Technical support from manufacturer was assured & weekend and night \\
& - Reliable delivery of bottles was assured & - Delivery of bottles was not always reliable \\
& - Expert with training in troubleshooting was available in & - No sustainable delivery for suitable glass bottles for \\
- Reduced, no daily manual inspection needed & self-prepared BHI broth could be established \\
- Incubation shortened to 5 days without final sub-culture & - Daily inspection for growth \\
& - False-positive signals were reduced (10/178, 5.6\%) & - Incubation for 10 days, final sub-culture recommended \\
& & - Often false-positive signals without subsequent growth of \\
& microorganisms $(88 / 219,40.2 \%)$
\end{tabular}

similar for automated and commercially available manual BC as we could not establish a reliable delivery chain for suitable glass bottles for self-prepared BHI broth for the increased request of BC.

\section{External Quality Control - Retesting of Isolates}

Of all 440 BC (including single automated and manual BC), 269 were positive $(61.1 \%)$. Of those 269 positive BC, 272 microorganisms could be recovered as in some $\mathrm{BC}$ multiple bacteria were found. However, not all isolates could be stored. Thus, in total, 228 isolates with identification at CHU-B were sent for retesting to Germany including duplicates recovered in corresponding automated and manual BC (Figure 3). Of those, 20 isolates could not be revived or identified. Identification using MALDI-TOF had to be repeated 45-times (36 isolates); in 6 cases, VITEK-2 was used for identification, but one isolate that had been identified as Klebsiella spp. could not be identified with automated identification tools (MALDI-TOF and VITEK ${ }^{\circledR}$ ). Isolates that had been identified as Salmonella Typhi were 


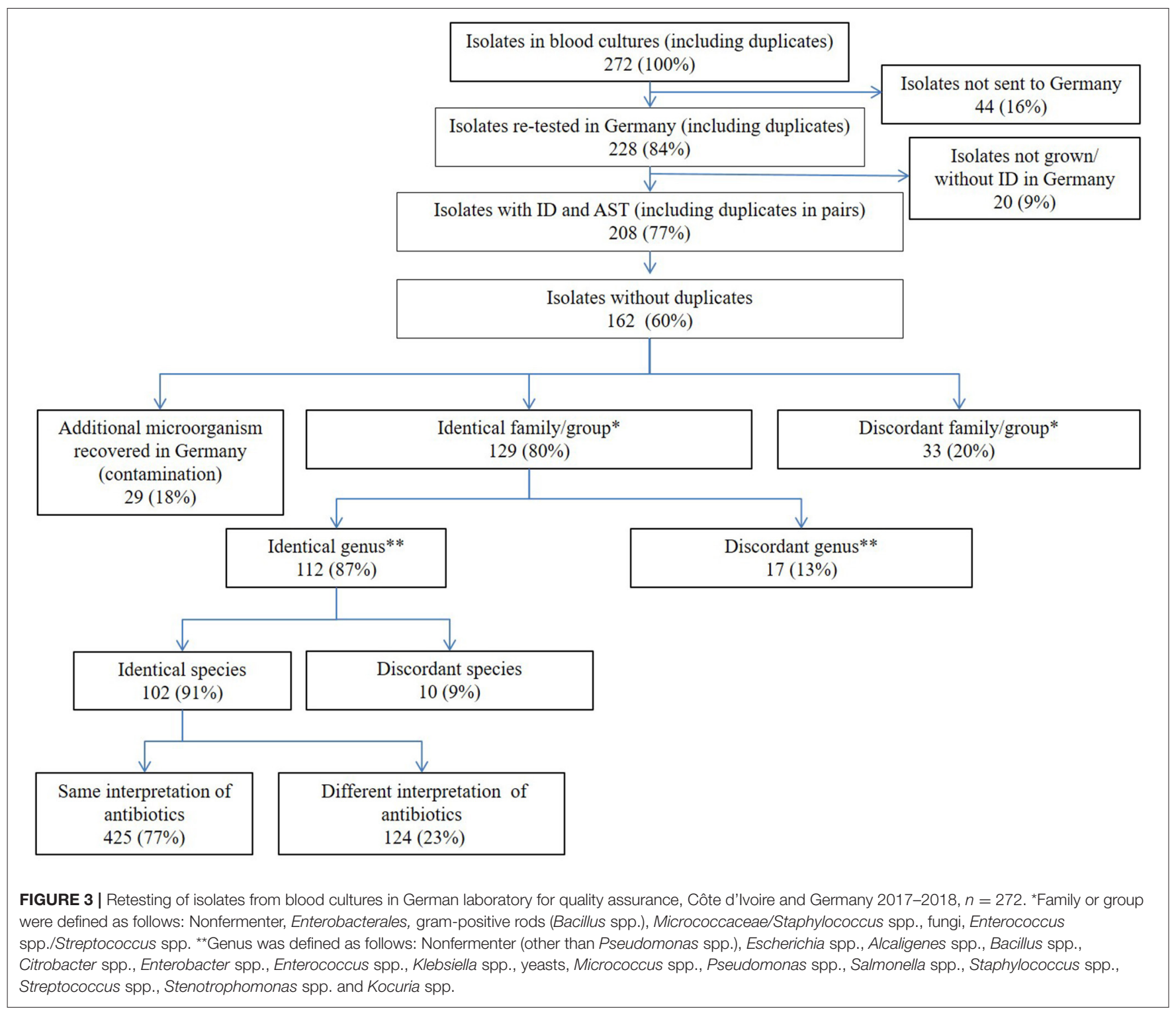

excluded from retesting $(n=1)$. In total, 208 isolates $(77 \%$ of all isolates sent for retesting) could be retested with identification and AST. Excluding duplicates from automated and manual $\mathrm{BC}$ pairs, 162 unique isolates were retested in Germany. In 29 cases, more microorganisms were found in the sample in Germany than detected at CHU-B. Of those 162 isolates, identification on family or group level was concordant in $79.6 \%$ $(n=129)$. Differences were mostly found in isolates identified as Staphylococcus spp. at CHU-B ( $n=16 / 62,25.8 \%)$. They were identified as non-fermenters $(8 / 16,50.0 \%)$ or Bacillus spp. $(5 / 16$, $31.3 \%)$ in retesting. In 112 isolates (69.1\%), the identification was concordant on genus level and in 102 isolates (63.0\%) concordant on species level. Of all Staphylococcus spp. identified at CHU-B, $67.7 \%(n=42 / 62)$ were concordant on species level in retesting, whereas $59.7 \%(n=40 / 67)$ of Enterobacterales were concordant on species level. Of those 102 isolates, AST interpretation was concordant in $77.4 \%$ of antibiotics $(n=425)$ in retesting.
Teicoplanin was concordant in $10 \%$ and vancomycin in $40 \%$ of tests. Here, the AST at CHU-B was more often non-susceptible than the AST in retesting. Eight of 10 (80.0\%) Staphylococcus aureus isolates with concordant species identification in retesting were concordant in methicillin-susceptibility or -resistance testing (MSSA or MRSA). One MSSA was identified as MRSA and one MRSA was identified as MSSA in retesting. Of 42 Enterobacterales isolates, $83.3 \%(n=35)$ were concordant in susceptibility to third-generation cephalosporins (cefotaxime, ceftriaxone or ceftazidime). Susceptibility to carbapenems in Gram-negative bacilli ( $n=43$ tests) was concordant in $86.0 \%$ ( $n=37$ ); of those, 36 belonged to Enterobacterales and one to Pseudomonas aeruginosa; three isolates (Enterobacter cloacaecomplex and Klebsiella pneumoniae) that were tested susceptible to meropenem at CHU-B were resistant upon retesting whereas three Enterobacterales isolates that were tested intermediate were susceptible to meropenem upon retesting. 


\section{DISCUSSION}

Our study showed that automated BC was superior to manual $\mathrm{BC}$ in this resource-limited setting at a University hospital in Côte d'Ivoire. The yield was higher and turnaround times were shorter in automated compared to manual BC. Automated BC enabled the laboratory to inform the clinicians $76 \mathrm{~h}$ earlier about first positive BC results than with manual BC. Furthermore, our study increased BC sampling from 24 to 63 BC per month. These findings are in accordance with other studies in resource-limited settings $(12,20)$. Additionally, the proportion of false-positive BC and therefore the workload could be reduced with automated BC significantly. Before considering the implementation of automated tools in resource-limited settings, the conditions, costs, and needs of automated vs. manual methods should be carefully considered according to the local setting. The proportion of $\mathrm{BC}$ with growth of a pathogen was high in both, automated and manual $\mathrm{BC}$, and above the targeted 5$15 \%$ (21). This could be an indicator that still too few BC were sampled and patients with blood stream infections were missed. Contamination of BC was high in automated and manual BC (48 and 43\%, respectively), although clinical training on good practice BC sampling supported our study. The proportion of contaminated BC was higher in our study than in comparable studies in resource-limited settings and above the targeted 3\% $(20,22-24)$. As most contaminants belonged to the typical flora of the skin, we assume that contamination occurred during sampling due to insufficient disinfection.

We found that half of the results of AST were concordant in automated and manual BC if the same pathogen was found. Differences affected several antibiotic classes. An internal quality control study in Switzerland found that even in highresource settings $14 \%$ of samples were not accurately retested in the same microbiological laboratory and 3\% of tested antibiotics were interpreted differently (25). By the time of the study, internal quality controls including sterility tests were in place, but commercially available quality control strains were not established.

For a good quality management system the implementation of external quality control programmes is essential $(13,26)$. As no external quality control was in place at CHU-B at the time of the study, we retested isolates in a German routine microbiology laboratory. The comparison of manual and automated methods for identification should be interpreted with caution as these use different principles. The identification of microorganisms from CHU-B posed difficulties for the automated tools in retesting and had to be retested several times or could not be identified at all. In some cases, more isolates were recovered in the samples upon retesting. The additionally recovered isolates could have been present but not discriminated at CHU-B or contamination occurred during storage or planting. In only $11 \%$, a different bacterial genus was identified in retesting. This highlights the good quality of manual identification despite the limited resources. Manual identification did not allow discriminating all bacteria to species level, but in resource-limited settings grouping of bacteria according to their clinical relevance might be reasonable and shorten turnaround time $(11,27)$. No systematic discordance between the identification tools could be identified. The differences might partly be explained by contamination or documentation error. Another reason might be that sheep blood was not available for the preparation of blood agar. Human blood discarded by blood bank had to be used instead. After the study, the laboratory successfully introduced self-prepared sheep blood agar sustainably. This was enabled by an expert exchange with other sub-Saharan countries. Different AST results were found in $23 \%$ of tested antibiotics upon retesting although the same interpretation reference was used predominantly. EUCAST had been implemented at CHU-B as this is a freely available reference (17). However, differences could partly be explained by different AST methods and references. If no EUCAST breakpoints for disk diffusion were available, the references from the Clinical and Laboratory Standards Institute (CLSI) were used at CHU-B. This could explain why teicoplanin and vancomycin were interpreted as more resistant at $\mathrm{CHU}-\mathrm{B}$ than in retesting. Inter-test variability should also be considered to explain some of the differences. The culture of microorganisms is influenced by many factors, e.g., culture media, inoculation of plate, incubation atmosphere and time or stochastic growth variability. We experienced that the provided EUCAST learning materials were very helpful to introduce EUCAST, but in part too sophisticated. Difficulties implementing EUCAST in resource-limited settings is especially hampered because defibrinated horse blood is not available for AST of fastidious bacteria. This has been published also by others and we recommend a low-resource adapted EUCAST version to overcome these obstacles $(4,28)$.

We asked clinicians to take blood for culture whenever they assumed a bloodstream infection, but we did not assess how well the recommendations were followed. To increase adherence and to avoid overloading of the laboratory capacities, we asked clinicians for one BC pair (automated and manual) only. If multiple BC were collected from one patient, these were counted as multiple cases. Anaerobic sub-culture was not available in the laboratory. For CO2-enriched cultures, a candle jar was used. This might explain why only few streptococci were found. Additionally, the attending microbiologist evaluated if the recovered bacteria were defined as contaminant or pathogen individually based on multiple criteria, not only identification. This might lead to different results.

Our study emphasizes that the implementation of automated tools such as BC incubation might be of value, but that good quality manual microbiological methods for identification and AST might not be inferior to automated processes. Before considering the implementation of automated tools, they should be adapted to the conditions and needs in resourcelimited settings as they are costly, require regular training and maintenance and are not adapted to the tropical climate and environment (11). In our study setting, the price for automated $\mathrm{BC}$ bottles was higher than for BC with self-prepared BHI broth. However, we could not establish a sustainable delivery of suitable, reusable glass bottles for the increased $\mathrm{BC}$ requests. Therefore, we had to purchase commercially available manual BC bottles, so the price did not differ much from the price of automated $\mathrm{BC}$ bottles. Moreover, the supply chain for automated BC bottles in our study setting was more reliable. The maintenance and 
technical support of automated BC is critical. Laboratory staff trained in troubleshooting for automated $\mathrm{BC}$ were available in the laboratory throughout our study period. All microbiological laboratory staff were trained in the loading and unloading of automated BC stepwise. The BacT/ALERT ${ }^{\circledR}$ 3D system was placed in a separate and clean room with air-conditioning. We did not experience power shortcuts with breakdown of the machine. For sustainable implementation of automated tools, the system has to be integrated in the laboratory strategy, funding beyond study periods has to be assured and therefore, all levels, including ministries of health, should be integrated in the process.

In expert interviews conducted in Africa and Asia, all interviewers listed environmental conditions as a significant challenge for BC processing (29). Further challenges are the different spectrum of "tropical" bacteria. Our study showed that automated tools might have difficulties identifying these bacteria. More important than implementing automated identification and AST tools are the implementation of good quality management systems including internal and external quality control (27). Laboratories face huge problems as necessary reagents for manual identification are not available anymore. To ensure good quality manual identification it is essential to maintain the production and delivery of these necessary reagents.

Our study was one step to implement and improve diagnostic stewardship at $\mathrm{CHU}-\mathrm{B}$. We were able to improve sampling and processing of $\mathrm{BC}$ in the laboratory. Diagnostic stewardship should be further strengthened and focus intensively on sampling, specimen transport and reporting of results. Continuous mentorship programmes including technical support and training should be maintained. Our study was an important step to include AST results in the local and national AMR surveillance and enables the comparison of $\mathrm{BC}$ results between different sites using either automated or manual BC.

\section{CONCLUSION}

Automated BC could be a valuable tool for the microbiological laboratory in resource-limited settings if the maintenance is assured and the staff is well-trained. Automated BC was superior to manual $\mathrm{BC}$ in sensitivity and timeliness. Mentorship to improve diagnostic stewardship further should focus on BC sampling and reporting of results. It is very important to implement and integrate good laboratory practices to improve manual microbiological methods for identification and AST and to ensure availability of necessary reagents. The implementation of automated tools should be decided individually according to economic considerations, number of samples processed, stable supply chain of consumables, and technical sustainability.

\section{DATA AVAILABILITY STATEMENT}

The datasets presented in this article are not readily available because the data will be made available upon reasonable request to interested researchers. Requests to access the datasets should be directed tolaera@rki.de.

\section{ETHICS STATEMENT}

The studies involving human participants were reviewed and approved by Charité University Ethics Committee Berlin, Germany (reference number: EA2/230/17) and the National Ethics Committee for Research in Abidjan, Côte d'Ivoire (reference number: 141/MHSP/CNER-km). Written informed consent from the participants' legal guardian/next of kin was not required to participate in this study in accordance with the national legislation and the institutional requirements.

\section{AUTHOR CONTRIBUTIONS}

AL, MN'G, FT, TE, KN, and CA-K contributed to conception and design of the study. AL, MN'G, FT, and CA-K implemented the study, generated and collected the data in Côte d'Ivoire. $\mathrm{RI}$ and JF generated and collected the data in Germany. KN, ST, FL, TE, and CA-K supervised the whole study and analysis. $\mathrm{AL}, \mathrm{KG}$, and JF organized the database. AL performed the data analysis and wrote the first draft of the manuscript. All authors critically reviewed the manuscript and approved the submitted version.

\section{FUNDING}

Robert Koch Institute received fundings through the Global Health Protection Programme (GHPP) of the German Federal Ministry of Health (grant number ZMVI1-2517-GHP-703) and from the German Federal Ministry of Education and Research (grant number 01KA1606, ANDEMIA).

\section{ACKNOWLEDGMENTS}

We are grateful to the laboratory and clinical personnel for their support and all their work for this study and to the management of CHU-B, Prof. Diané Bamourou. We thank HansPeter Blank for the double entry of the data. We would also like to thank the whole team of the Postgraduate Training for Applied Epidemiology at RKI, Katharina Alpers, Irina Czogiel, and especially Christian Winter for their continuous support throughout the study. We thank Benedikt Zacher for his statistical support and Susanne Koehler, Grit Schubert, and Essia Belarbi for their help to realize the study. Finally, we thank Michael Mueller, head of MVZ Labor 28, for his support.

\section{SUPPLEMENTARY MATERIAL}

The Supplementary Material for this article can be found online at: https://www.frontiersin.org/articles/10.3389/fmed. 2021.627513/full\#supplementary-material

Supplementary Figure 1 | Identification flow chart for manual identification of Gram-positive cocci and Gram-negative bacilli used during the study period, CHU-B, Côte d'Ivoire 2017-2018. 


\section{REFERENCES}

1. Reddy EA, Shaw AV, Crump JA. Community-acquired bloodstream infections in Africa: a systematic review and meta-analysis. Lancet Infect Dis. (2010) 10:417-32. doi: 10.1016/S1473-3099(10)70072-4

2. World Health Organization. Antimicrobial Resistance: Global Report on Surveillance. Geneva (2014).

3. World Health Organization. Global Action Plan on Antimicrobial Resistance. WHA68/2015/REC/1, Annex 3, Geneva (2015).

4. Ombelet S, Ronat JB, Walsh T, Yansouni CP, Cox J, Vlieghe E, et al. Clinical bacteriology in low-resource settings: today's solutions. Lancet Infect Dis. (2018) 18:e248-58. doi: 10.1016/S1473-3099(18)30093-8

5. World Health Organization. Global Antimicrobial Resistance Surveillance System: Manual for Early Implementation. Geneva (2015).

6. World Health Organization. Diagnostic Stewardship - A Guide to Implementation in Antimicrobial Resistance Surveillance Sites. Geneva (2016).

7. Rohner P, Pepey B, Auckenthaler R. Comparison of BacT/Alert with Signal blood culture system. J Clin Microbiol. (1995) 33:313-7. doi: 10.1128/JCM.33.2.313-317.1995

8. Kirn TJ, Mirrett S, Reller LB, Weinstein MP. Controlled clinical comparison of BacT/alert FA plus and FN plus blood culture media with BacT/alert FA and FN blood culture media. J Clin Microbiol. (2014) 52:83943. doi: 10.1128/JCM.03063-13

9. Hellinger WC, Cawley JJ, Alvarez S, Hogan SF, Harmsen WS, Ilstrup DM, et al. Clinical comparison of the isolator and BacT/Alert aerobic blood culture systems. J Clin Microbiol. (1995) 33:1787-90. doi: 10.1128/JCM.33.7.1787-1790.1995

10. Anagonou SY, Akpona S, Josse R, Massougbodji A, Sadeler BC. Les isolement de bacteries dans les hémocultures au laboratoire de bacteriologie du C.N.H.U.-Cotonou (1987-1990). Méd d'Afrique Noire. (1993) 40:614-9.

11. Ombelet S, Barbe B, Affolabi D, Ronat JB, Lompo P, Lunguya O, et al. Best practices of blood cultures in low- and middle-income Countries. Front Med. (2019) 6:131. doi: 10.3389/fmed.2019.00131

12. Elantamilan D, Lyngdoh WV, Banik A, Khyriem AB, Bhattacharyyn P, Rajbongshi J, et al. Comparative evaluation of conventional (manual) blood culture system and BacT/ALERT 3D (automated) blood culture system in a Tertiary care hospital. Scholars J Appl Med Sci. (2017) 5:544-9. doi: 10.1159/000092186

13. Barbé B, Yansouni CP, Affolabi D, Jacobs J. Implementation of quality management for clinical bacteriology in low-resource settings. Clin Microbiol Infect. (2017) 23:426-33. doi: 10.1016/j.cmi.2017.05.007

14. Akoua-Koffi C, Tia H, Plo JK, Monemo P, Cissé A, Yao C, et al. Epidemiology of community-onset bloodstream infections in Bouaké, central Côte d'Ivoire. New Microbe New Infect. (2015) 7:100-4. doi: 10.1016/j.nmni.2015.06.009

15. Muller-Schulte E, Tuo MN, Akoua-Koffi C, Schaumburg F, Becker SL. High prevalence of ESBL-producing Klebsiella pneumoniae in clinical samples from central Cote d'Ivoire. Int J Infect Dis. (2020) 91:2079. doi: 10.1016/j.ijid.2019.11.024

16. Akoua-Koffi C. Rapport Brut D’activités 2016 Laboratoire BactériologieVirologie. Centre Hospitalier Universitaire Bouaké, Laboratoire de Bactrériologie-Virologie, Bouaké (2017).

17. The European Committee on Antimicrobial Susceptibility Testing. Breakpoint Tables for Interpretation of MICs and Zone Diameters. Version 7.1 (2017). Available online at: http://www.eucast.org (accessed February 12, 2020)
18. The European Committee on Antimicrobial Susceptibility Testing. Breakpoint Tables for Interpretation of MICs and Zone Diameters. Version 8.1 (2018). Available online at: http://www.eucast.org (accessed February 12, 2020)

19. Christiansen TB, Lauritsen JM. EpiData - Comprehensive Data Management and Basic Statistical Analysis System. Odense: EpiData Association (2010).

20. Karam El-Din A-ZA, Mohamed MA, Gad WH, Lotfy GS. Prevalence of microbial pathogens in blood cultures: an etiological and histopathological study. J Taibah Univ Sci. (2010) 3:23-32. doi: 10.1016/S1658-3655(12)60017-X

21. Leber A. Blood Cultures. Clinical Microbiology Procedures Handbook. 4th ed. Washington, DC: ASM Press (2016).

22. Hill PC, Onyeama CO, Ikumapayi UN, Secka O, Ameyaw S, Simmonds N, et al. Bacteraemia in patients admitted to an urban hospital in West Africa. BMC Infect Dis. (2007) 7:2. doi: 10.1186/1471-2334-7-2

23. Abrahams MS, Whitelaw AC, Orth H. Time for a culture change? Suboptimal compliance with blood culture standards at a district hospital in Cape Town. South Afr Med J. (2015) 105:1039-43. doi: 10.7196/SAMJ.2015.v105i12. 9442

24. Obeng-Nkrumah N, Labi AK, Addison NO, Labi JE, Awuah-Mensah G. Trends in paediatric and adult bloodstream infections at a Ghanaian referral hospital: a retrospective study. Ann Clin Microbiol Antimicrob. (2016) 15:49. doi: 10.1186/s12941-016-0163-Z

25. Scherz V, Durussel C, Greub G. Internal quality assurance in diagnostic microbiology: a simple approach for insightful data. PLoS ONE. (2017) 12:e0187263. doi: 10.1371/journal.pone.0187263

26. World Health Organization. Laboratory Quality Management System Handbook. Geneva (2011).

27. Jacobs J, Hardy L, Semret M, Lunguya O, Phe T, Affolabi D, et al. Diagnostic bacteriology in district hospitals in Sub-Saharan Africa: at the forefront of the containment of antimicrobial resistance. Front Med. (2019) 6:205. doi: 10.3389/fmed.2019.00205

28. Cusack TP, Ashley EA, Ling CL, Roberts T, Turner P, Wangrangsimakul $\mathrm{T}$, et al. Time to switch from CLSI to EUCAST? A Southeast Asian perspective. Clin Microbiol Infect. (2019) 25:782-5. doi: 10.1016/j.cmi.2019. 03.016

29. Dailey PJ, Osborn J, Ashley EA, Baron EJ, Dance DAB, Fusco D, et al. Defining system requirements for simplified blood culture to enable widespread use in resource-limited settings. Diagnostics. (2019) 9:10. doi: 10.3390/diagnostics9010010

Conflict of Interest: RI and JF were employed by the company MVZ Labor_28 GmbH.

The remaining authors declare that the research was conducted in the absence of any commercial or financial relationships that could be construed as a potential conflict of interest.

Copyright (c) 2021 von Laer, N’Guessan, Touré, Nowak, Groeschner, Ignatius, Friesen, Tomczyk, Leendertz, Eckmanns and Akoua-Koffi. This is an open-access article distributed under the terms of the Creative Commons Attribution License (CC BY). The use, distribution or reproduction in other forums is permitted, provided the original author(s) and the copyright owner(s) are credited and that the original publication in this journal is cited, in accordance with accepted academic practice. No use, distribution or reproduction is permitted which does not comply with these terms. 